\title{
QUELQUES EFFETS MAGNÉTIQUES DANS DES SUPRACONDUGTEURS
}

\author{
P. PINCUS,
}

Physique des Solides, Faculté des Sciences d'Orsay, 91-Orsay.

Résumé. - On discute les effets sur les températures et les champs critiques des forces magnétiques qui ne sont pas invariantes par inversion du temps. Par exemple, on considère les impuretés paramagnétiques, champs externes, films magnétiques superposés, etc.

Abstract. - We discuss the effects of magnetic forces which are not time reversal invariant on the transition temperature and critical fields of superconductors. For example, we consider paramagnetic impurities, external fields, superimposed magnetic films, etc.

I. Introduction. - Dans un supraconducteur, les électrons sont conjugués deux à deux dans des états de moment et spin opposés par inversion du signe du temps pour former des « paires de Cooper ». Donc des forces qui ne sont pas invariantes par inversion du temps ont une tendance à briser ses paires. Les interactions magnétiques agissent de cette manière. Leur effet est de baisser la température critique $T_{\mathrm{c}}$ et de créer un champ magnétique critique au-dessus duquel le matériau devient normal. On va rappeler ces effets dans quelques cas différents.

London a montré que l'existence des courants superfluides implique un diamagnétisme parfait dans un supraconducteur massif, c'est-à-dire :

$$
B=H+4 \pi M=0
$$

(pour un cylindre avec $H$ parallèle à l'axe pour lequel il n'y a pas de champ démagnétisant). Un champ

TABLEAU I ( $\left.{ }^{\mathbf{1}}\right)$

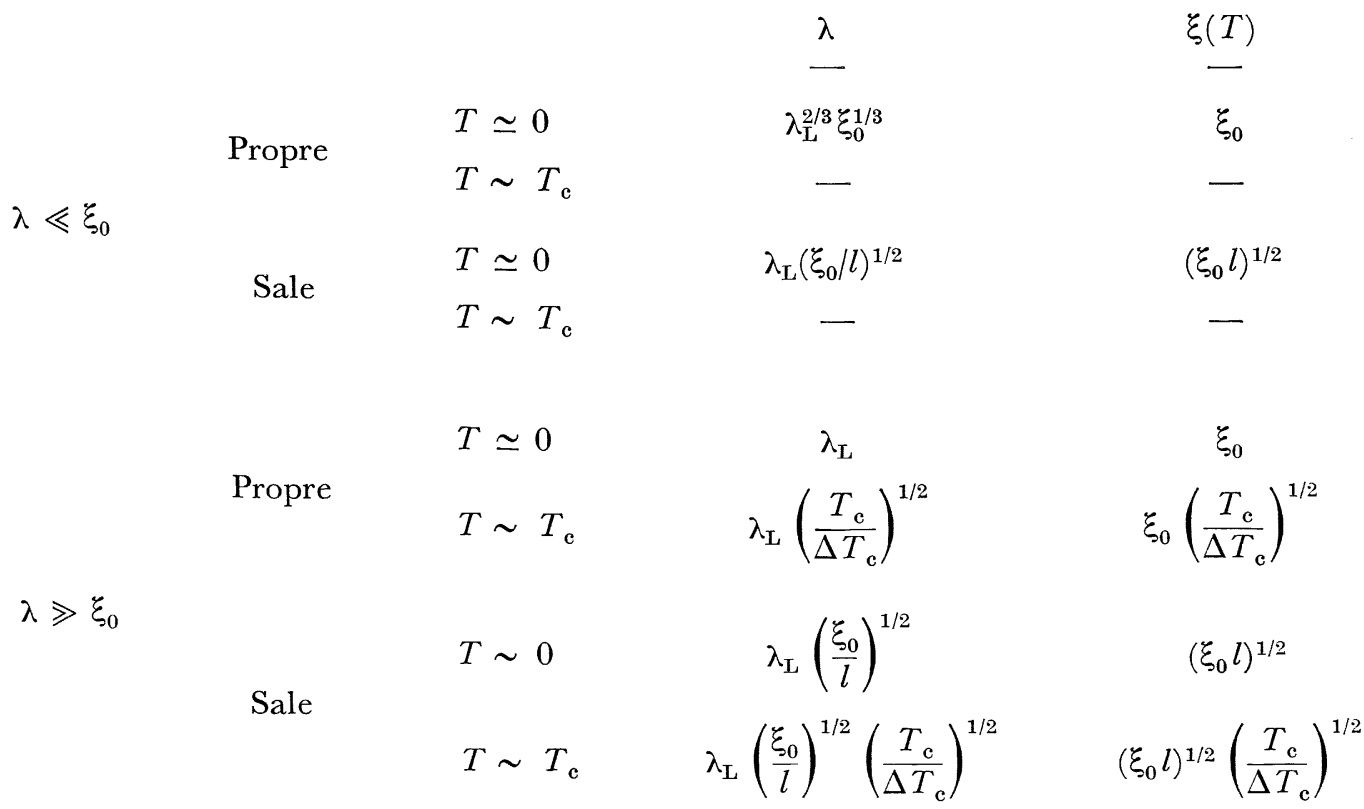

(1) Pour les coefficients numériques, voir P. G. DE GENnES, "Superconductivity of Metals and Alloys " [1]. 
externe pénètre seulement une distance microscopique $\lambda$ (profondeur de pénétration). Donc, dans l'état supraconducteur, il y a une perte d'énergie magnétique par rapport à l'état normal. Par ailleurs, on gagne une énergie due au couplage en « paires».

En comparant les énergies libres (par unité de volume) de ces deux cas, on trouve :

$$
\Delta F=F_{\mathrm{n}}-F_{\mathrm{s}}=\frac{H^{2}}{8 \pi}-\frac{H_{\mathrm{c}}^{2}}{8 \pi}
$$

où :

$$
\frac{H_{\mathrm{c}}^{2}}{8 \pi}=\frac{n(0)}{2} \Delta^{2}
$$

$(n(0)$ est la densité d'états au niveau de Fermi dans l'état normal et $\Delta(T)$ est la bande d'énergie interdite qui dépend de la température; $\Delta(T)=1,76 k_{B} T_{\mathrm{c}}$ quand $T \rightarrow 0) ; H=H_{\mathrm{c}}(T)$ donne la limite de séparation des phases $\mathrm{N}$ et $\mathrm{S}$.

Avant de discuter les effets magnétiques, il est commode d'introduire plusieurs longueurs caractéristiques de l'état supraconducteur. Nous avons déjà cité la profondeur de pénétration $\lambda$.

Par ailleurs, on a :

$-\xi_{0}$ - rayon d'une « paire de Cooper » $=\frac{\hbar v_{\mathrm{f}}}{\pi \Delta(0)}$
$\left(v_{\mathrm{f}}=\right.$ vitesse de Fermi $)$,

- $\xi(T)$ - la distance caractéristique sur laquelle $\Delta$ relaxe vers sa valeur d'équilibre s'il est perturbé en un point,

- $l$ - libre parcours moyen résiduel pour les électrons au niveau de Fermi,

- $\lambda_{\mathrm{L}}=\frac{m c^{2}}{4 \pi n e^{2}}$ - profondeur de pénétration de London ( $n=$ densité des électrons).

Le tableau I donne plusieurs valeurs de ces longueurs caractéristiques en fonction de la température $(T=0$, $\left.T \simeq T_{\mathrm{c}}\right)$ et suivant que le matériau est « propre » $\left(l \gg \xi_{0}\right)$ ou « sale » $\left(l \ll \xi_{0}\right)$.

II. Systèmes homogènes. - A. DEux EsPÈcEs (I et II). - Considérons une région normale $\left(T<T_{\mathrm{c}}\right)$ dans un supraconducteur en champ magnétique. La taille minimale d'une telle région est de l'ordre de $\xi(T)$. La perte d'énergie de couplage en paires dans cette région est alors :

$$
\sim \frac{H_{\mathrm{c}}^{2}}{8 \pi} \xi(\tau)
$$

Le gain d'énergie magnétique est :

$$
\sim \frac{H_{\mathrm{e}}^{2} \delta}{8 \pi}
$$

$(\delta \sim \xi(T)$ pour $\xi(T)>\lambda ; \delta \sim \lambda$ pour $\lambda>\xi(T))$.

Gette région normale est donc stable pour : $\frac{H_{\mathbf{c}}^{2}}{8 \pi} \delta>\frac{H_{\mathbf{c}}^{2}}{8 \pi} \xi(T)$. Pour $\xi(T)>\lambda$ (supraconducteur dit de type I), cette condition donne $H>H_{\text {c }}$, ce qui implique qu'il n'y a pas de pénétration du flux en dessous de $H_{\mathrm{c}}$. Autrement, pour $\xi(T)<\lambda$ (type II), on trouve que le flux peut pénétrer. Cette pénétration s'effectue sous la forme des lignes de tourbillons d'Abrikosov. Le champ critique pour l'entrée de la première ligne de flux $\left(H_{\mathrm{c}_{1}}\right)$ est :

$$
H_{\mathrm{c}_{1}} \sim H_{\mathrm{c}}(\xi(T) / \lambda)<H_{\mathrm{c}} .
$$

Pour $H_{\mathrm{c}} \sim 10^{2}$ Oe, $\xi \simeq 10^{-6} \mathrm{~cm}, \lambda \simeq 10^{-5} \mathrm{~cm}$, $H_{\mathrm{c}_{1}} \simeq 10 \mathrm{Oe}$.

Chaque ligne porte un quantum de flux :

$$
\varphi_{0}=\frac{c h}{2 e} \simeq 2 \times 10^{-7} \mathrm{G} \times \mathrm{cm}^{2} .
$$

L'induction de l'échantillon est $B=N \varphi_{0}, N=$ nombre de lignes par unité de surface.

La transition vers l'état normal se produit quand toutes les régions normales se touchent, c'est-à-dire lorsque $N \sim \xi^{-2}$.

Cette condition donne le champ critique supérieur $H_{\mathrm{c}_{2}} \sim \varphi_{0} / \xi^{2}=H_{\mathrm{c}}(\lambda / \xi)>H_{\mathrm{c}}$. Pour $H_{\mathrm{c}} \sim 10^{2}$ Oe, $\lambda \sim 10^{-5} \mathrm{~cm}, \xi \simeq 10^{-6} \mathrm{~cm}, H_{\mathrm{c}_{2}} \sim 10^{3} \mathrm{Oe}$.

Néanmoins, dans quelques cas favorables, on peut arriver à $H_{\mathrm{c}_{2}} \sim 10^{5} \mathrm{Oe}\left(\mathrm{Nb}_{3} \mathrm{Sn}, \mathrm{V}_{3} \mathrm{Si}\right)$.

Sur la figure 1, on a tracé des courbes idéales d'aimantation pour ces deux types de comportement.

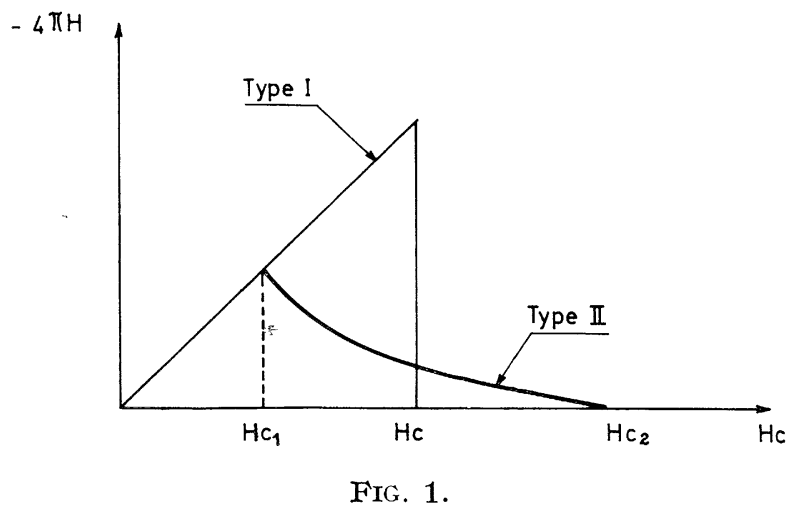

Pour les champs critiques très élevés $\left(\sim 10^{5} \mathrm{Oe}\right)$, on n'a plus le droit de négliger la susceptibilité de Pauli comme nous l'avons fait jusqu'ici. En fait, dans la condition où le couplage spin-orbite est petit, la susceptibilité de spin des paires tend vers zéro. Donc, il faut ajouter dans l'énergie libre un terme correspondant :

$$
\Delta F=\frac{H^{2}}{8 \pi}+\chi_{\mathrm{p}} \frac{H^{2}}{8 \pi}-\frac{H_{\mathrm{c}}^{2}}{8 \pi}
$$

$\left(\chi_{\mathrm{p}} \simeq \mu_{B}^{2} n(0)\right.$ est la susceptibilité de Pauli). L'état supraconducteur est instable par rapport à l'état normal quand $\chi_{\mathrm{p}} H^{2}>H_{\mathrm{c}}^{2}$; c'est-à-dire $\mu_{B} H>1,76 k_{B} T_{\mathrm{c}}$ (pour $T_{\mathrm{c}} \sim 10 \mathrm{o}, H_{\mathrm{p}} \sim 10^{5} \mathrm{Oe}$ ).

$H_{\mathrm{p}}$ est une limite supérieure de champ critique. 
Hake a étudié cet effet en détail. Le couplage spinorbite diminue l'effet de $\chi_{\mathrm{p}}$ parce que la susceptibilité des paires devient finie.

B. Impuretés PARAmAgnétiQues. - Ici, on considère le couplage entre le spin localisé $\mathbf{S}$ de l'impureté et les spins des électrons de bande $\mathbf{s}$ de la forme :

\section{JS.S}

( $J \sim 1 \mathrm{eV}$ est une énergie d'échange).

Ce couplage agit avec signes apposés sur les deux électrons d'une « paire de Cooper». Qualitativement, ce couplage se traduit par un temps de vie $\tau$, pour une telle « paire ». La température critique $T_{\mathrm{c}}$ est donc diminuée :

$$
\delta\left(k_{B} T_{\mathrm{c}}\right) \sim \hbar / \tau
$$

Dans ce cas, $\hbar \tau^{-1} \sim \frac{J^{2}}{E_{F}} x \quad(x$ est la concentration atomique des impuretés).

On trouve $\delta T_{\mathrm{c}} \sim 10^{3} x$. L'effet est très important.

G. Impuretés non magnétiques en Ghamp. - Pour les petits échantillons (taille caractéristique $d<\lambda$ ) où le champ appliqué est presque uniforme, les électrons subissent l'effet d'un potentiel vecteur aléatoire à cause de la diffusion par les impuretés, c'est-à-dire que le moment orbital change brutalement. Ici, on trouve :

$$
\tau^{-1} \sim \frac{H^{2} d^{2} l v_{\mathrm{f}}}{\varphi_{0}^{2}}
$$

III. Systèmes inhomogènes. - A. EFFETS DE PROXIMITÉ. - Considérons un sandwich F-S-F où F est un matériau ferromagnétique et $\mathrm{S}$ un film mince $\left(d<\xi_{0}, l\right)$ supraconducteur. Si les deux lames $\mathrm{F}$ sont aimantées parallèlement, il existe un champ d'échange uniforme qui donne un $\delta T_{\mathrm{c}} \sim J\left\langle S_{\mathrm{z}}\right\rangle(a / d)$ (a est une distance interatomique, $\left\langle S_{z}\right\rangle$ est la moyenne d'aimantation).

Typiquement, on trouve $\delta T_{\mathrm{c}} \sim 0,5 \mathrm{oK}$. Par contre, si $F_{1}$ et $F_{2}$ sont alignés antiparallèlement, le champ moyen est nul et il n'y a pas d'effet au premier ordre en $J$.

B. InGlusions ferromagnétiques. - Considérons des petits grains ferromagnétiques dans un supraconducteur. Si le contact électronique est bon, il y a un effet de proximité. Si le contact est mauvais (couches oxydées), il reste un effet dû aux champs dipolaires.
1. Bon contact. - Il faut considérer deux cas limites suivant que le rayon du grain est petit ou grand par rapport à $k^{-1}$, la profondeur de pénétration de paires de Cooper dans les régions ferromagnétiques. Cette distance est donnée par :

$$
k^{-1} \sim\left\{\begin{array}{ll}
\hbar v_{\mathrm{f}} / J & \leftrightarrow \ll \text { Propre » } \\
\left(\hbar v_{\mathrm{f}} l / J\right)^{1 / 2} & \leftrightarrow \text { 《Sale } »
\end{array} \sim\right.
$$

Pour $k b \gg 1$, le paramètre d'ordre est négligeable dans les grains avec $\Delta=0$ en surface. Le résultat est très semblable à celui des impuretés paramagnétiques avec :

$$
\tau^{-1} \sim x b v_{\mathrm{f}} l
$$

où $x$ est le nombre des grains par unité de volume.

Remarquons que $\tau$ est indépendant de l'aimantation. Au contraire, dans le cas $b k \ll 1$, le paramètre d'ordre est presque constant et l'effet des grains est d'ajouter un champ d'échange uniforme comme dans la section précédente. On trouve d'une façon analogue :

$$
\delta T_{\mathrm{c}} \sim J\left\langle S_{\mathrm{z}}\right\rangle b^{3} x .
$$

2. Mauvais contact. - Si l'amas magnétique est soit isolant, soit entouré par une couche d'oxyde, le contact électronique est mauvais et le seul effet des grains est d'imposer un champ dipolaire agissant sur les « paires de Cooper ». Dans un supraconducteur de type II, on trouve une répartition du champ anisotrope autour de chaque sphère qui varie $(r \gg \lambda)$ comme :

$$
h \sim M_{0}(b / r)(b / \lambda)^{2} \mathrm{e}^{-r / \lambda} .
$$

Ge champ donne un couplage dipolaire entre grains qui est différent dans l'état normal et supraconducteur. A cause de la grande portée de l'interaction dipolaire (dans l'état normal), cette différence d'énergie dépend de la forme de l'échantillon par l'intermédiaire du coefficient de désaimantation.

Si tous les grains sont alignés, on trouve (pour un cylindre) :

$$
\frac{\delta T_{\mathrm{c}}}{T_{\mathrm{c}}} \sim M / H_{\mathrm{c}}(0)
$$

où $M$ est l'aimantation totale ( 10 Oe si $1 \%$ de l'échantillon est ferromagnétique) et $H_{\mathrm{c}}(0)$ est le champ critique thermodynamique à $T=0$.

$$
\text { Pour } M \sim 10 \mathrm{Oe}, H_{\mathrm{c}} \sim 10^{3} \mathrm{Oe}, \frac{\delta T_{\mathrm{c}}}{T_{\mathrm{c}}} \sim 10^{-2} \text {. }
$$

\section{BIBLIOGRAPHIE}

\section{Général :}

[1] DE GENNES (P. G.), "Superconductivity of Metals and Alloys ", Benjamin, 1961.

FETTER (A.) et Hobenberg (P.), "Theory of type II Superconductors ", Parks, Superconductivity, Dekker (1969), New York.
Paramagnétisme de Pauli :

Chandrasekhar (B. S.), Appl. Phys. Lett., 1962, 1, 7 .

Clogston (A. M.), Phys. Rev. Lett., 1962, 1, 66. Werthamer (N. R.), Helfand (E.) et HohENBERG (P. C.), Phy's. Rev., 1966, 147, 295. 
Champ d'échange:

Th. : Sarma (G.), J. Phys. Chem. Sol., 1963, 24, 1029.

Couplage entre couches ferromagnétiques :

Th. : DE GENNES (P. G.), Phys. Lett., 1966, 23, 10.

Exp. : Deutscher (G.) et Meunier (F.), Phys, Rev. Lett., 1969, 22, 395.
Inclusions ferromagnétiques:

Exp. : RosenblatTT (J.), Hauser (J.), ValetTte (C.) et Burger (J. P.), L. T. XI.

Th. : Hurauli (J. P.) et Pincus (P.), "Low Temp. Phys. ", 1969, 1, 129.

Dobrosavlevic (L.), J. Physique, 1969, 30, 589. 